\title{
Multiphysics Modelling of Ultrasonic Melt Treatment in the Hot-Top and Launder during Direct-Chill Casting: Path to Indirect Microstructure Simulation
}

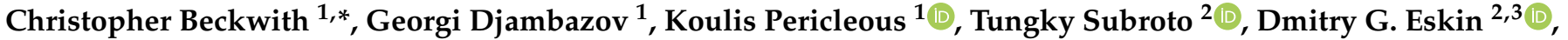 \\ Dan Roberts ${ }^{4}$, Ivan Skalicky ${ }^{4}$ and Iakovos Tzanakis ${ }^{5,6}$ (D)
}

1 Computational Science and Engineering Group, University of Greenwich, 30 Park Row, London SE10 9LS, UK; G.Djambazov@greenwich.ac.uk (G.D.); k.pericleous@greenwich.ac.uk (K.P.)

2 Brunel Centre for Advanced Solidification Technology (BCAST), Brunel University London, Uxbridge UB8 3PH, UK; tungky.subroto@brunel.ac.uk (T.S.); Dmitry.Eskin@brunel.ac.uk (D.G.E.)

3 Department of Mathematical Physics, Faculty of Physics and Engineering, Tomsk State University, 634050 Tomsk, Russia

4 Constellium UTC, Brunel University London, Uxbridge UB8 3PH, UK; dan.roberts@constellium.com (D.R.); ivan.skalicky@constellium.com (I.S.)

5 Faculty of Technology, Design and Environment, Oxford Brookes University, Oxford OX33 1HX, UK; itzanakis@brookes.ac.uk

6 Department of Materials, University of Oxford, Oxford OX1 3PH, UK

Citation: Beckwith, C.; Djambazov, G.; Pericleous, K.; Subroto, T.; Eskin, D.G.; Roberts, D.; Skalicky, I.;

Tzanakis, I. Multiphysics Modelling of Ultrasonic Melt Treatment in the Hot-Top and Launder during Direct-Chill Casting: Path to Indirect Microstructure Simulation. Metals 2021, 11, 674. https://doi.org/ $10.3390 /$ met11050674

Academic Editors: Anders E. W. Jarfors and Shusen Wu

Received: 20 March 2021

Accepted: 16 April 2021

Published: 21 April 2021

Publisher's Note: MDPI stays neutral with regard to jurisdictional claims in published maps and institutional affiliations.

Copyright: (c) 2021 by the authors. Licensee MDPI, Basel, Switzerland. This article is an open access article distributed under the terms and conditions of the Creative Commons Attribution (CC BY) license (https:/ / creativecommons.org/licenses/by/ $4.0 /)$.
* Correspondence: c.beckwith@greenwich.ac.uk

\begin{abstract}
This study concerns the numerical simulation of two competing ultrasonic treatment (UST) strategies for microstructure refinement in the direct-chill (DC) casting of aluminium alloys. In the first, more conventional, case, the sonotrode vibrating at $17.3 \mathrm{kHz}$ is immersed in the hop-top to treat the sump melt pool, in the second case, the sonotrode is inserted between baffles in the launder. It is known that microstructure refinement depends on the intensity of acoustic cavitation and the residence time of the treated fluid in the cavitation zone. The geometry, acoustic field intensity, induced flow velocities, and local temperature are factors which affect this treatment. The mathematical model developed in this work couples flow velocity, acoustics modified by cavitation, heat transfer, and solidification at the macroscale, with Lagrangian refiner particles, used to determine: (a) their residence time in the active zones, and (b) their eventual distribution in the sump as a function of the velocity field. This is the first attempt at using particle models as an efficient, though indirect, alternative to microstructure simulation, and the results indicate that UST in the launder, assisted with baffle separators, yields a more uniform distribution of refining particles, avoiding the strong acoustic streaming jet that, otherwise, accompanies hot-top treatment, and may lead to the strong segregation of refining particles. Experiments conducted in parallel to the numerical studies in this work appeared to support the results obtained in the simulation.
\end{abstract}

Keywords: ultrasonic processing; DC casting; cavitation; Lagrangian tracking

\section{Introduction}

In the casting of aluminium alloys, the improvement of microstructure by the reduction of grain size is important due to multiple benefits, ranging from improved mechanical properties, to increased resistance to hot tearing [1]. A number of methods have been tested, including the addition of a grain refiner [2], the application of an ultrasonic field through the use of a mechanical sonotrode [3], an alternating current (AC) induction coil [4], or the combination of an AC induction coil with a background DC field [5]. The efficiency of ultrasonic melt treatment (UST) is attributed to the onset of the formation and explosive collapse of bubbles in the melt (cavitation), due to large pressure oscillations [6]. Extreme temperatures $(>10,000 \mathrm{~K})$ [7] and pressures ( $>400 \mathrm{MPa})$ [8] occur at the centre of these 
collapsing bubbles, which triggers nucleation on, and fragmentation of, the solidification substrates (refining particles), and ultimately refines the microstructure. The two primary mechanisms driving grain refinement due to UST have previously been reviewed in [3], and can be explained by: (i) the activation of latent (e.g., $\mathrm{Al}_{2} \mathrm{O}_{3}$ ) or intentionally added (e.g., $\mathrm{TiB}_{2}$ ) non-metallic inclusions, which then enhance heterogeneous nucleation; and (ii) fragmentation of primary intermetallics (e.g., $\mathrm{Al}_{3} \mathrm{Zr}$ ). If UST is applied during directchill (DC) casting by immersing the mechanical sonotrode directly inside the hot-top, the acoustic jet can additionally cause primary crystals of aluminium to be fragmented. This is a common approach in the DC casting process [9], however a weakness of this approach is that with current implementations, effective treatment is restricted by the active zone being confined to a narrow region under the sonotrode. An alternative idea which could help upscale this process is to place the sonotrode further upstream in the launder. The addition of partitions, or baffles, to regulate the flow can then be used to increase residence time and allow more of the aluminium alloy to be effectively processed. In our previous work, this approach was investigated in pilot scale experiments in the DC casting of an AA6XXX alloy with $\mathrm{Zr}$ addition [10], and we showed that grain refinement can also be observed when processing is carried out in the launder, primarily driven by the activation/fragmentation of particles, which then act as nucleation sites in the solidifying volume. Ideally, a cross section of the resulting ingot should have a uniform and fine grain structure throughout the billet. In this work, we extend this idea by numerically comparing the effectiveness of the two approaches to see which one is more advantageous in terms of potential structural refinement. A coupled numerical method is developed by using a finite element solver for the nonlinear Helmholtz cavitation model [11], coupled to turbulent buoyant fluid flow computed in a custom OpenFOAM solver, which incorporates an enthalpy method [12] for the computation of the solidifying sump. The fully 3D model runs in parallel and simulates for the first time the combined process of DC casting, UST, and launder melt feeding. The use of a Helmholtz cavitation model is validated by comparing the results to the frequency domain signal obtained by applying a fast Fourier transform (FFT) to the pressures obtained, using a time domain Caflisch equation solver [13]. A Lagrangian tracking algorithm is then used to predict the distribution of the transported refining particles, and some experimental validation is provided to correlate this with the observed grain refinement from actual billets.

\section{Experimental Setup}

DC casting of AA6XXX series aluminium alloy billets was carried out in the Advanced Metal Casting Centre (AMCC) of the Brunel Centre of Advanced Solidification Technology (BCAST). The diameter of the produced billets was $152 \mathrm{~mm}$, and the casting was done with a hot-top. More information regarding the DC-casting set up can be found elsewhere [10]. All billets were cast to a length of approximately $1 \mathrm{~m}$ at a casting speed of $140 \mathrm{~mm} / \mathrm{min}$ (corresponds to around $2.5 \mathrm{~L} / \mathrm{min}$ melt flow-rate). The billets were cast at a temperature of $668 \pm 5{ }^{\circ} \mathrm{C}$. The experimental setup of the ultrasonic melt treatment (UST)-DC casting is illustrated in Figure 1a. A $5 \mathrm{~kW}$ water-cooled magnetostrictive transducer was used, coupled with a $\mathrm{Nb}$ sonotrode with a $20 \mathrm{~mm}$ working diameter operated at $17.3 \mathrm{kHz}$ at a $3.5 \mathrm{~kW}$ power ( $40 \mu \mathrm{m}$ amplitude peak-to-peak). Three billets were cast for this study. The first billet was cast using a conventional DC casting setup (without UST), the second billet was cast with UST carried out in the DC casting hot-top (Figure 1a), and the third was cast with UST performed in the DC casting launder (Figure 1b). For the third billet, to compare the effect between UST and non-UST conditions, the ultrasonic transducer was only activated once a steady state regime had been reached and the first half part of the billet had been cast (approximately $0.5 \mathrm{~m}$ of the billet length). 


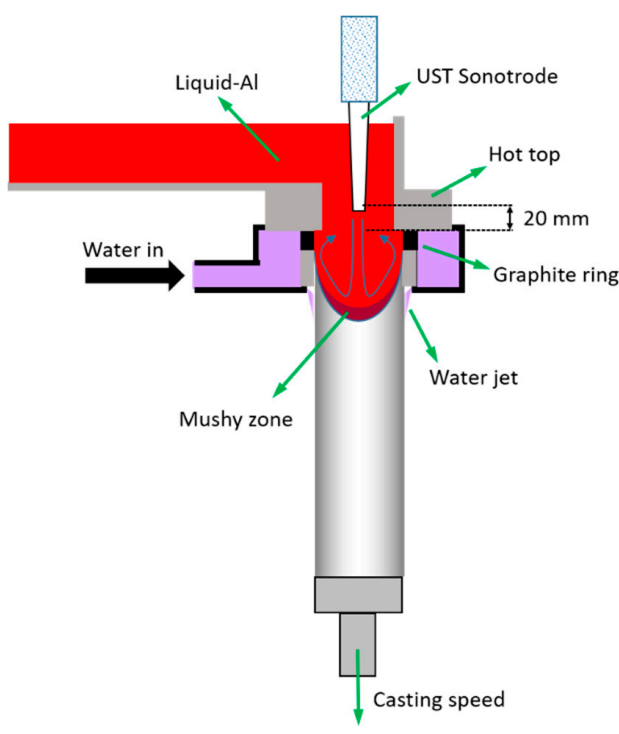

(a)

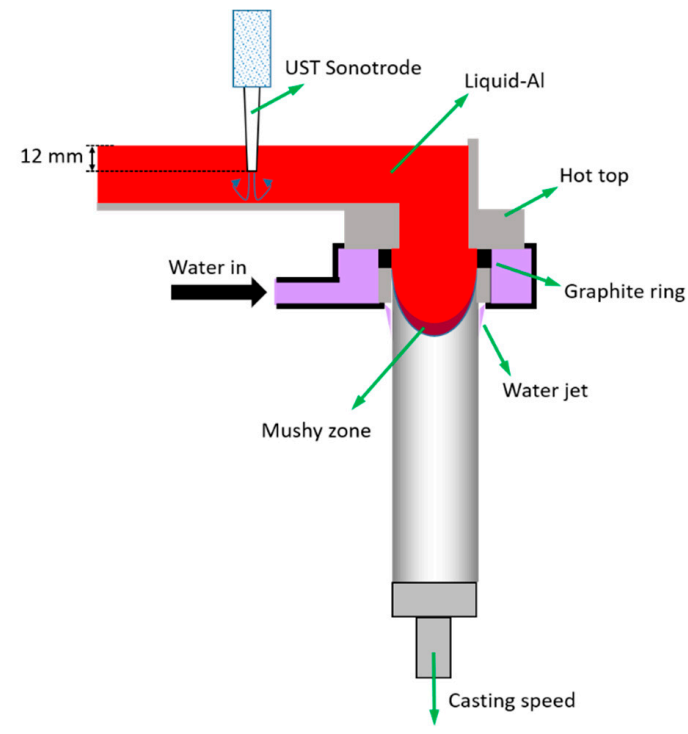

(b)

Figure 1. Illustration of DC casting setup and different locations of ultrasonic melt treatment (UST). (a) On the DC casting hot-top, and (b) on the DC casting launder.

The effect of UST on microstructure was verified through grain size analysis. For microstructure observation, samples were cut from different parts across the billet, from the centre to the surface. The samples were ground and polished, and subsequently anodized in $5 \mathrm{wt} . \% \mathrm{HBF}_{4}$ in water solution (Barker's reagent) using $20 \mathrm{VDC}$, and then examined under a Carl Zeiss (Oberkochen, Germany) Axioscope A1 optical microscope with polarized light. The average grain size was measured using the linear intercept method taken randomly from microscope images and then statistically analysed. The chemical composition of the alloys (Table 1) differed slightly between the first two billets and the third one. There was some AlTiB grain refiner added to the first and second billets. While the third billet had $0.2 \mathrm{wt} . \% \mathrm{Zr}$ added as a grain-refiner. It was reported that $\mathrm{Al}_{3} \mathrm{Zr}$ is a very potent substrate for aluminium grains upon UST, as efficient as $\mathrm{TiB}_{2}$ [12]. For the purpose of simulation, we considered the particles that provide substrates for aluminium as equivalent irrespective of their nature. Since the focus was to compare the effectiveness of structure refinement at different locations of UST treatment, we used the normalised grain size value at different UST locations with the maximum value considered as unity value.

Table 1. Chemical composition (wt.\%) of the alloy obtained through optical emission spectroscopy (OES).

\begin{tabular}{cccccccc}
\hline Al & Si & $\mathbf{C u}$ & $\mathbf{M g}$ & $\mathbf{M n}$ & $\mathbf{Z r}$ & $\mathbf{F e}$ & $\mathbf{T i}$ \\
\hline Balance & 0.8 & 0.73 & 0.68 & 0.48 & 0.21 & 0.2 & 0.04 \\
\hline
\end{tabular}

\section{Numerical Methods}

Numerical simulations of UST treatment in a DC casting launder with settings (e.g., launder geometry, sonotrode tip geometry, and immersion level, etc.) imitating the experimental conditions were carried out to provide guidelines on the optimisation of UST in the melt flow by using flow control partitions. A nonlinear Helmholtz-type model [9] was especially developed to compute the acoustic pressure field, accounting for the effect of cavitation. This is given in Equation (1), where $P$ represents the complex acoustic pressure field, and $k_{m}^{2}$ is a modified wave number given by Equation (2).

$$
\nabla^{2} P+k_{m}^{2} P=0
$$




$$
k_{m}^{2}=\left(\frac{\omega}{c}\right)^{2}-\left(\frac{\mathcal{A}(P)}{|P|}+i \frac{\mathcal{B}(P)}{|P|}\right),
$$

where $\omega$ is the angular frequency and $c=4600 \mathrm{~m} / \mathrm{s}$ is the speed of sound in liquid aluminium. The dissipation functions $\mathcal{A}$ and $\mathcal{B}$ take the same form as in [11]. In the previous work [10] a weighting function was included to aid convergence, but this was not necessary here since the discontinuity at the Blake threshold was naturally smooth when evaluating the attenuation coefficients dependent on the local pressure rather than using a reference value. Then, the real part of $\omega / k_{m}$ describes the change in speed of sound, and the imaginary part of $k_{m}$ describes the attenuation due to the existence of inertially cavitating bubbles. To model the bubble activity, the Keller-Miksis (KM) approach [14] is used, as given in Equation (3).

$$
\left(1-\frac{\dot{R}}{C}\right) R \ddot{R}+\frac{3}{2} \dot{R}^{2}\left(1-\frac{\dot{R}}{3 c}\right)=\frac{1}{\rho_{l}}\left(1+\frac{\dot{R}}{c}+\frac{R}{c} \frac{d}{d t}\right)\left[p_{l}-p(t)\right]
$$

Here $p_{l}$ represents the liquid pressure at the liquid gas interface. The pressure $p(t)=p_{0}(1-A \sin (\omega t))$ accounts for the atmospheric and acoustic pressures. In Lebon et al. [15], the amplitude parameter $A$ was chosen to be 2.4, matching that of the expected readings from experiments in liquid aluminium [16]. However, in this work individual bubble simulations were performed at a range of pressures from $A=1$ up to $A=4$, and the computed coefficients were referred to as a function of the local pressure in the Helmholtz equation. This more closely follows the method of Trujillo [17]. $\mathcal{A}$ and $\mathcal{B}$ can be calculated using Equation (4), which considers only the change in void fraction over the last acoustic period.

$$
\mathcal{A}=-\frac{\rho_{l} \omega^{2}}{\pi} \int_{0}^{2 \pi} \frac{\partial \beta}{\partial \tau} \sin \tau d \tau, \mathcal{B}=-\frac{\rho_{l} \omega^{2}}{\pi} \int_{0}^{2 \pi} \frac{\partial \beta}{\partial \tau} \cos \tau d \tau
$$

where $\beta$ is the void fraction and can be calculated using:

$$
\beta=\frac{4}{3} \pi R^{3} N
$$

where $N$ is the number of bubbles. For the fluid flow simulation, an OpenFOAM solver was developed using "buoyantPimpleFoam" (a combination of the PISO and SIMPLE algorithms) as a base. Solidification is modelled using a single continuum approach with the solid region acting as a momentum sink. The continuity and momentum equations are given in:

$$
\begin{gathered}
\nabla \cdot v=0 \\
\rho_{0} \frac{\partial v}{\partial t}+\rho_{0} \nabla \cdot(v v)=-\nabla p+\mu_{0} \nabla^{2} v+S_{b}+S_{d}+F_{s}
\end{gathered}
$$

where $\rho_{0}$ and $\mu_{0}$ are the fluid density and the dynamic viscosity, $S_{b}$ represents the buoyancy term given in Equation (8), and $S_{d}$ is the Carman-Kozeny momentum sink term that forces the fluid velocity to the background velocity $v_{r e f}$, as given by Equation (9). $\boldsymbol{F}_{s}$ is an additional term accounting for acoustic streaming and has the form $\boldsymbol{F}_{\boldsymbol{s}}=\nabla\left(\rho v_{a} \bar{\otimes} v_{a}\right)$, where $v_{a}$ is the acoustic velocity. The simulation was run until the flow reached steady state.

$$
\begin{gathered}
S_{b}=\rho_{0} g \beta_{T}\left(T-T_{r e f}\right) \\
S_{d}=-C \frac{\left(1-g_{l}\right)^{2}}{g_{l}^{3}}\left(v-v_{r e f}\right)
\end{gathered}
$$

where $C$ is the mushy zone constant, and $v_{\text {ref }}$ is the background velocity field, which is 0 in the launder, and the casting velocity in the DC caster. $S_{d}$ in Equation (9) is 0 in the liquid region but acts as a strong damping term as the melt fraction approaches zero, forcing the 
solid velocity to equal the casting velocity. Turbulence is included in the model, using the $\mathrm{k}$-Omega-SST turbulence model. The system is closed by the energy balance, as given in Equation (10).

$$
\rho c_{p} \frac{\partial T}{\partial t}+\rho c_{p} \nabla \cdot(v T)=\nabla \cdot(k \nabla T)-\rho_{0} L_{f}\left[\frac{\partial g_{l}}{\partial t}+\nabla \cdot\left(v g_{l}\right)\right]
$$

where $c_{p}$ is the specific heat, $T$ is the temperature, and $k$ is the thermal conductivity. $L_{f}$ in this equation accounts for the latent heat of fusion, and $g_{l}$ is the volume fraction of liquid. In the slurry, the effective dynamic viscosity $\mu_{\text {eff }}$ can be calculated from the Stefanescu formula $[18,19]$ given in Equations (11) and (12), where $\mu_{l}$ is the liquid viscosity, $f_{s}$ is the solid fraction, and $f_{c}$ is the dendrite coherency point, chosen to be 0.3 .

$$
\begin{gathered}
\mu_{\text {eff }}=\mu_{l}\left(\frac{1}{1-F_{\mu} f_{s} / f_{c}}\right)^{2} \\
F_{\mu}=0.5-(1 / \pi) \tan ^{-1}\left(100\left(f_{s}-f_{c}\right)\right)
\end{gathered}
$$

A summary of all the material properties used for liquid aluminium is given in Table 2. The heat transfer across a solid boundary can be approximated by the Fourier boundary condition $k \frac{\partial T}{\partial x}=q$, where $k$ represents the material conductivity. For the water spray, the heat flux is often computed directly using a forced-convection heat transfer equation $[15,20]$, but the resulting term can be approximated as being a linear function depending on the average temperature $\bar{T}$ between the surface of the billet and the bulk fluid [21]. Accounting for the effect of nucleate boiling above a critical point $q_{c}=3910 \Delta T^{2.16}$ the final heat transfer coefficient takes the form in Equation (13).

$$
h_{c}= \begin{cases}{\left[-1.67 \times 10^{5}+704 \bar{T}\right] \cdot Q^{\prime 1 / 3},} & \text { if } q_{c} \geq q_{i} \\ {\left[-1.67 \times 10^{5}+704 \bar{T}\right] \cdot Q^{\prime 1 / 3}+\frac{20.8\left(\Delta T_{x}\right)^{3}}{\Delta T},} & \text { if } q_{c}<q_{i}\end{cases}
$$

Table 2. Model properties of liquid aluminium. Properties obtained from [15].

\begin{tabular}{cc}
\hline Casting velocity $\left(\mathrm{m} \mathrm{s}^{-1}\right)$ & 0.0023 \\
\hline Inlet temperature $(\mathrm{K})$ & 1013.15 \\
\hline Liquidus temperature $(\mathrm{K})$ & 929.2 \\
\hline Solidus temperature $(\mathrm{K})$ & 757.4 \\
\hline Latent Heat $\left(\mathrm{J} \mathrm{kg}^{-1}\right)$ & $375,696.0$ \\
\hline Density $\left(\mathrm{kg} \mathrm{m}^{-3}\right)$ & 2375 \\
\hline Speed of sound $\left(\mathrm{m} \mathrm{s}^{-1}\right)$ & 4600 \\
\hline Thermal expansion coefficient $\left(\mathrm{K}^{-1}\right)$ & $2.3 \times 10^{-5}$ \\
\hline Kinematic viscosity $\left(\mathrm{m}^{2} \mathrm{~s}^{-1}\right)$ & $5.5 \times 10^{-7}$ \\
\hline
\end{tabular}

At the free surface, the loss of heat due to surface radiation is given by Equation (14), where $\epsilon=0.3$ is the surface emmisivity, and $\sigma=5.6708 \times 10^{-8}$ is the Stefan-Boltzmann constant.

$$
\nabla \mathrm{T}=\frac{\epsilon \sigma\left(T_{\mathrm{amb}}^{4}-T^{4}\right)}{k}
$$

While the nucleating particles like $\mathrm{TiB}_{2}$ or $\mathrm{Al}_{3} \mathrm{Zr}$ particles are small, they are prone to sediment and do not flow perfectly with the bulk fluid, meaning that a massless particle formulation is not appropriate. Apart from their slightly higher density, these particles are also plate-like in morphology, so a non-spherical drag model is needed and the method of [22] was used here. Ultrasonic processing of Al-Zr alloys showed a wide range of resul- 
tant refined particles, dependent on the processing temperature [23]. The fragmentation of these particles is not yet modelled and can be considered for future work. In this study, the density of nucleating particles was assumed to be $4140 \mathrm{~kg} \mathrm{~m}^{-3}$ [24] with a non-spherical drag model coefficient of $\phi=0.512$. The distribution and concentration of these particles can be linked to a reduction of grain size by the fact that they act as nucleation sites, giving clues as to how the microstructure might be affected. Lagrangian particle tracking via the discrete element method (DEM) was used to determine the location of nucleating particles in the final billet, as affected by the passage through regions of active cavitation. In previous work [25], we discussed the possibility of different launder configurations also affecting the total processing time, and found that there was only a small difference between those that were tested. For this reason, only one partition configuration was tested here, and the residence time of particles was compared against those processed in the hot-top.

\section{Results}

\subsection{Bubble Simulation}

Equations (1)-(3) are solved assuming a uniform initial distribution of $5 \mu \mathrm{m}$ hydrogen bubbles in a periodic acoustic field operating at a frequency of $17.3 \mathrm{kHz}$. The system of nonlinear equations is solved using the "DifferentialEquations.jl" Julia package, with the Tsitouras 5/4 Runge-Kutta algorithm used for time stepping [26]. Coefficients $\mathcal{A}$ and $\mathcal{B}$ were calculated from Equation (4), with the integrals taking the time average solution for the final acoustic cycle. The material properties for a hydrogen bubble are given in Table 3 [15].

Table 3. Hydrogen bubble properties [15].

\begin{tabular}{cc}
\hline Surface Tension $\left(\mathrm{N} \mathrm{m}^{-1}\right)$ & 0.860 \\
\hline Vapour pressure $(\mathrm{Pa})$ & 0 \\
\hline Specific Heat Capacity $\left(\mathrm{J} \mathrm{kg}^{-1} \mathrm{~K}^{-1}\right)$ & 717 \\
\hline Bulk Temperature $(\mathrm{K})$ & 1013.15 \\
\hline Ambient bubble radius $R_{0}(\mathrm{~m})$ & $5 \times 10^{-6}$ \\
\hline
\end{tabular}

The simulation was run for a maximum of 50 acoustic cycles, and cases that failed to converge to a stable harmonic solution were interpolated with a piecewise spline. Coefficients for the range $A=1$ to 4 are given in Figure 2. The vertical dotted line at approximately $A=2.177$ corresponds to the Blake threshold, after which the nonlinear formulation causes a significant increase in the attenuation coefficients, in line with that of previous work [15].

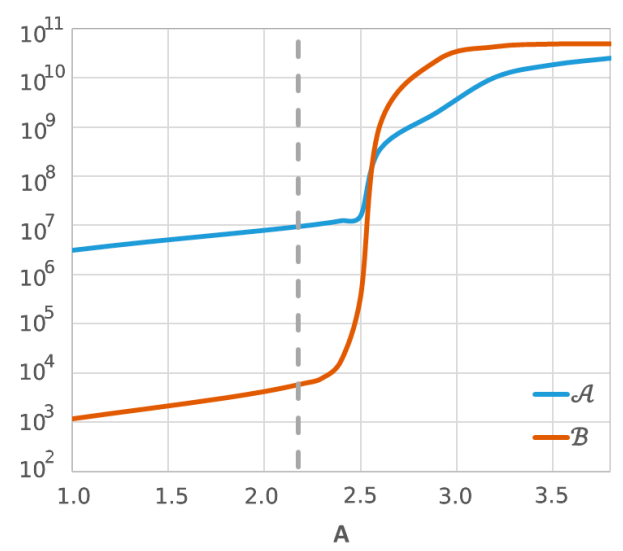

Figure 2. Change in nonlinear coefficients $\mathcal{A}$ and $\mathcal{B}$ as a function of the change in input amplitude, $\mathrm{A}$. Coefficients increase dramatically above the Blake threshold. 


\subsection{Casting Simulation}

Full 3D simulations were performed, first using COMSOL Multiphysics (version 5.2a, COMSOL Inc., Stockholm, Sweden.) to calculate the acoustic field, and then the results were converted to OpenFOAM format to calculate the turbulent fluid flow. The OpenFOAM solver described in Section 3 was implemented in OpenFOAM 7 (OpenFOAM Foundation, London, UK). The material boundary conditions used in the fluid flow simulation are given in Table 4. The inlet temperature was chosen to match the temperature recorded in the launder experiments. The simulation was run for a total of $300 \mathrm{~s}$ and took a total run time of approximately $4 \mathrm{~h}$ on a Ryzen 3600 CPU (AMD, California, USA) to complete, in addition to the $1 \mathrm{~h}$ needed to precompute the acoustic field. This makes the model a good option for uncertainty quantification studies where speed is of importance.

Table 4. Fluid flow model boundary conditions.

\begin{tabular}{|c|c|}
\hline \multicolumn{2}{|c|}{ P_rgh } \\
\hline Inlet & TotalPressure uniform 0 \\
\hline Outlet & ZeroGradient \\
\hline Free Surface, Hot-Top, Graphite, Mold & fixedFluxPressure uniform 0 \\
\hline Launder, Water, Sonotrode & fixedFluxPressure uniform 0 \\
\hline \multicolumn{2}{|c|}{$\mathbf{P}$} \\
\hline All & Calculated; \\
\hline \multicolumn{2}{|c|}{$\mathbf{U}$} \\
\hline Inlet & pressureInletOutletVelocity \\
\hline Outlet & fixedValue uniform (0-uCast 0 ) \\
\hline Launder, Water, Graphite, Mold, Sonotrode & noSlip \\
\hline Free Surface, Hot Top & Slip \\
\hline \multicolumn{2}{|c|}{$\mathbf{T}$} \\
\hline Inlet & fixedValue uniform 1034 \\
\hline Outlet, Free Surface, Hot Top, Launder & zeroGradient \\
\hline Water, Mold, Graphite & Fixed Gradient $=(h / k e)\left(T-T_{\text {inf }}\right)$ \\
\hline \multicolumn{2}{|l|}{ Free Surface, Hot Top } \\
\hline \multicolumn{2}{|c|}{ Alpha1 } \\
\hline Inlet & 1 \\
\hline $\begin{array}{l}\text { Outlet, Free Surface, Hot Top, Sonotrode, } \\
\text { Launder, Mold, Graphite }\end{array}$ & zeroGradient \\
\hline
\end{tabular}

To generate the complex geometry, the cfMesh cartesianMesh mesh generator was used, with a surface mesh generated using Salome. Coefficients $\mathcal{A}$ and $\mathcal{B}$ were precomputed over the range $1-4 x$ atmospheric pressure, with piecewise splines used to interpolate intermediate values. The acoustic pressure was then calculated from Equation (1), assuming that the acoustic pressure field can be approximated by a time harmonic solution. To verify the assumption that considering only the driving frequency is sufficient, full timedependent modelling was performed for the launder case. It was based on the Caflisch model of acoustic cavitation [11], and an efficient, in terms of computational time, implementation was achieved following a practical assumption about the effect of the cavitation bubble dynamics on the ultrasound field [27]. An FFT analysis of this time dependent result is shown in Figure 3a, and the computed raw pressure signals in Figure 3b. From the FFT analysis, the driving frequency was observed to dominate over the higher harmonics, validating the use of a harmonic model. While it is possible that the application of UST 
could lead to visible surface vibrations, the effect is minimal compared to the main acoustic field, and no noticeable surface vibrations were detected in experiments. For this reason, the free surface is assumed to be a fixed sound-soft boundary.

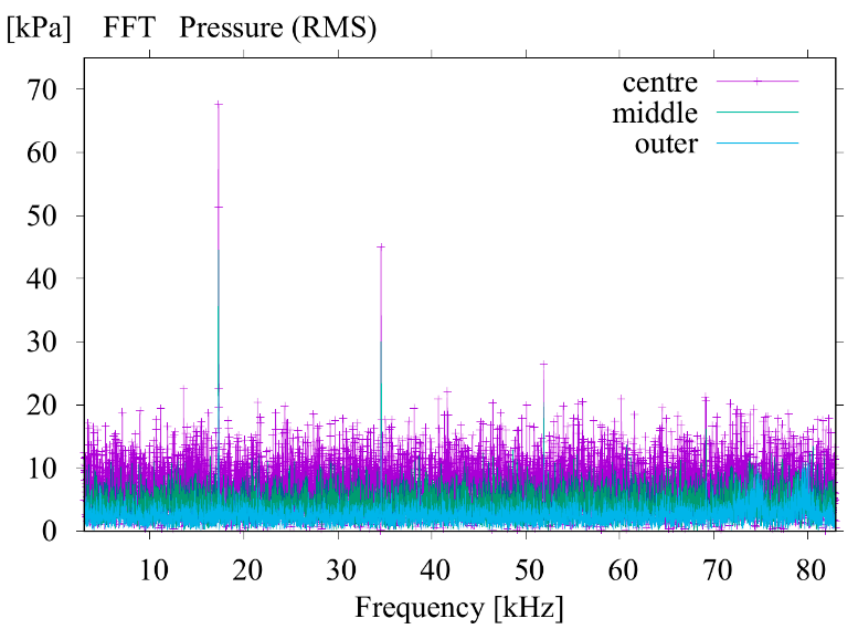

(a)

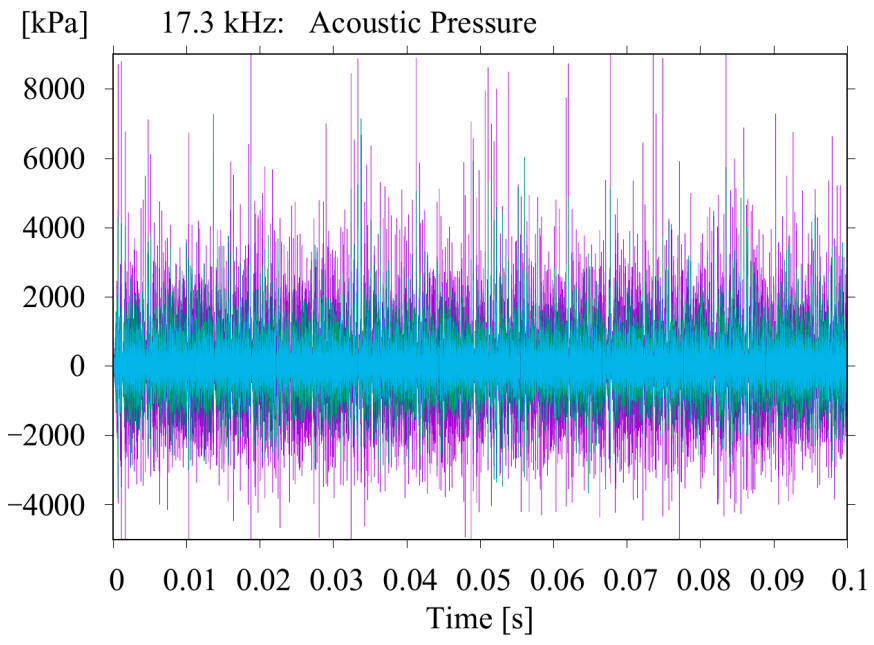

(b)

Figure 3. (a) FFT frequency response from a Caflisch cavitation model with the sonotrode positioned in the launder, and (b) the time series data at three nodal locations.

The time harmonic pressure distribution for processing in the launder is given in Figure $4 \mathrm{a}$, and in the hot-top in Figure $4 \mathrm{~b}$. Peak pressures under the sonotrode are seen to be comparable in both cases, but the launder processing sustains a larger region around the Blake threshold pressure $(\sim 217 \mathrm{kPa})$ [10]. A comparison of the amplitudes in a straight vertical line below the centre of the sonotrode is given in Figure 4c. The acoustic waves are attenuated rapidly in the region just under the sonotrode, forming the primary cavitation region. The acoustic energy is dissipated in the form of thermal and viscous losses, as well as acoustic radiation. It is in this region that large bubble structures develop and contribute to the effect known as acoustic shielding [28].
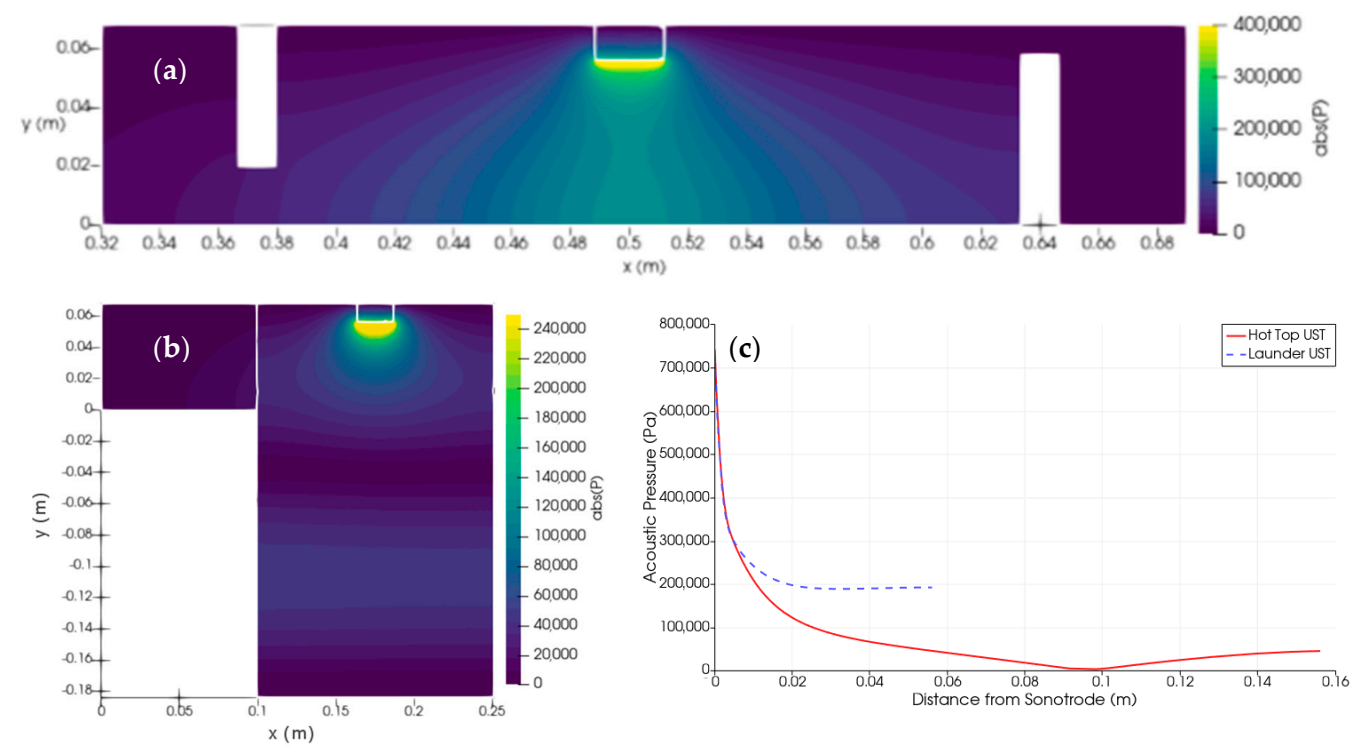

Figure 4. Acoustic pressure distribution in the launder (a) and the hot-top (b) a comparison of the pressure observed as a function of the vertical distance from the sonotrode tip is given in (c). 
Differences in the acoustic field also affected the magnitude of the induced streaming volumetric force $\left(\mathrm{N} \mathrm{m}^{-3}\right)$, and vector plots of the induced forces are given in Figure 5. The red highlighted region visible under the sonotrode in both the launder and the hot-top corresponds to the region with acoustic pressures above the Blake threshold $(\sim 217 \mathrm{kPa})$. The induced force is several orders of magnitude higher in a narrow layer directly under the sonotrode, and the magnitude of the force is comparable in both cases.

(a)

(b)

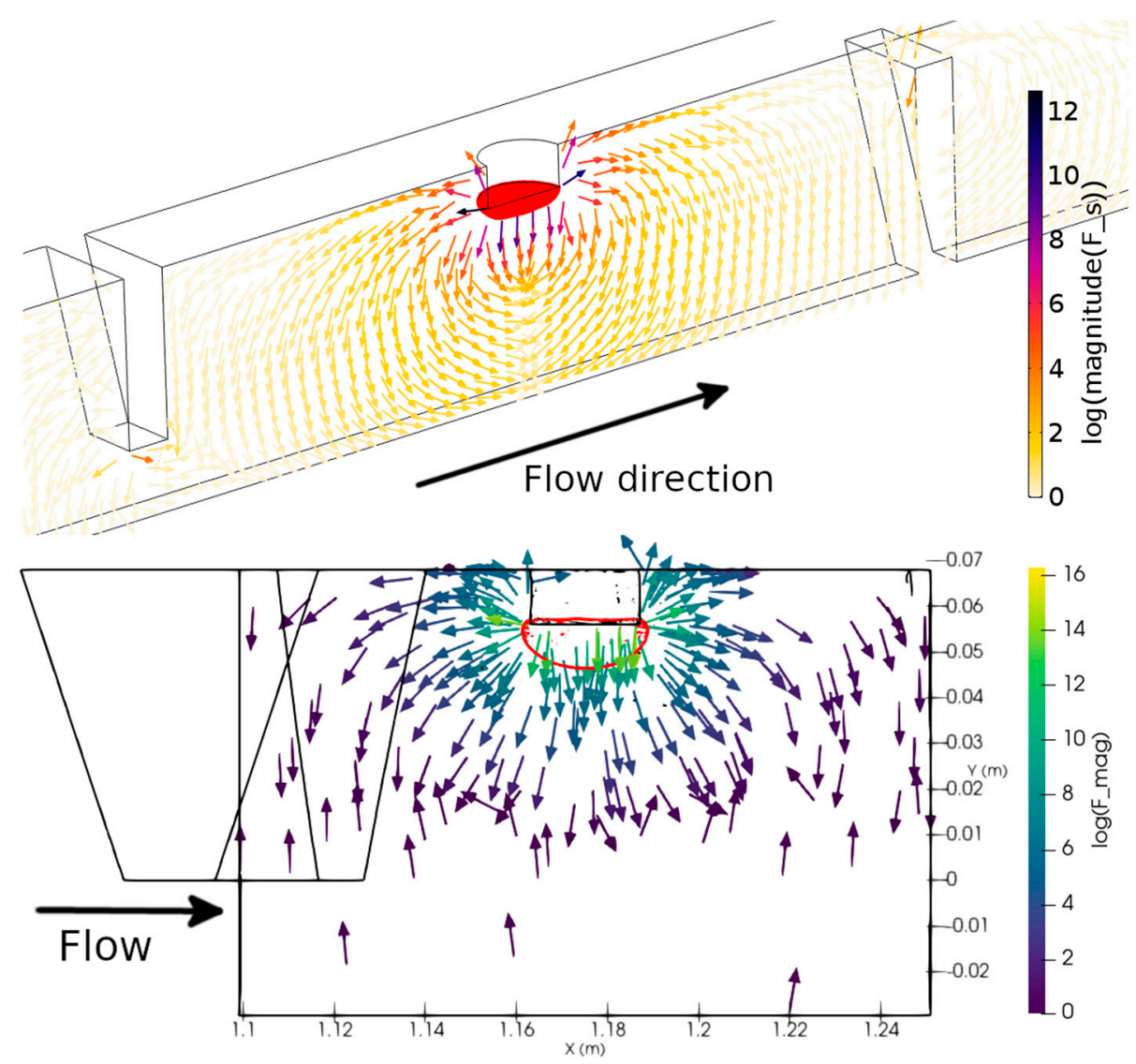

Figure 5. Magnitude of the induced acoustic streaming force when applying UST in the launder (a), and in the hot-top (b).

When UST is applied in the launder, the flow in the hot-top is dominated by the side melt feeding velocity, as can be seen in Figure $6 \mathrm{~b}$. This feeding results in an asymmetric flow profile, which could not be revealed in earlier 2D axisymmetric simulations [15]. This is due to the feeding velocity pushing against the flow expected from natural convection. When UST was applied in the hot-top directly, the results agreed more closely with the axisymmetric model used in [15]. In this case, the velocity field is dominated by the acoustic streaming jet and not the flow inlet velocity. This can be seen in Figure $7 \mathrm{~b}$, with streaming velocities of the order $0.5 \mathrm{~m} / \mathrm{s}$. Streaming velocities in the hot-top were found be lower than when processing in the launder, where streaming velocities of the order $1 \mathrm{~m} / \mathrm{s}$ were found (Figure 8c). The transition zone from the fully liquidus state to the fully solidus state is compressed in the centre of the domain, resulting in a wider transition zone near the surface of the billet. The compressed transition region increased the temperature gradient, a result which agrees with that of [15]. This can clearly be observed by the temperature contours in Figure 7a. It has been suggested that these high thermal gradients could lead to increased hot tearing risk [29], despite reducing the overall tearing risk due to grain refinement [1]. 


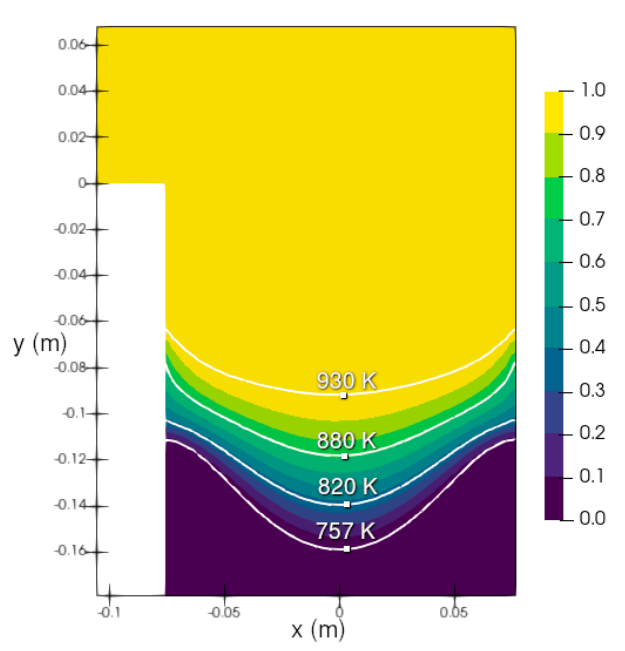

(a)

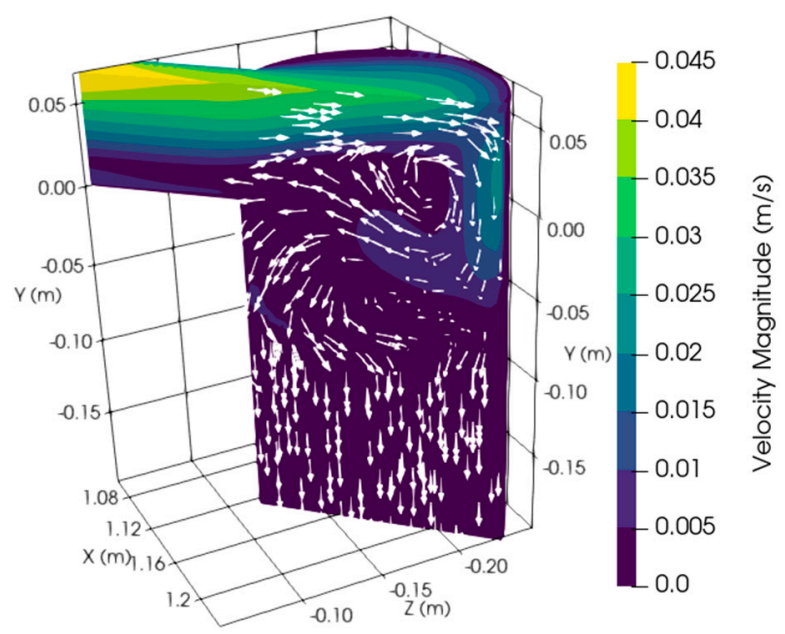

(b)

Figure 6. Resulting solid fraction and temperature profile (a), and fluid flow velocity magnitude (b) in the billet when applying UST in the launder.

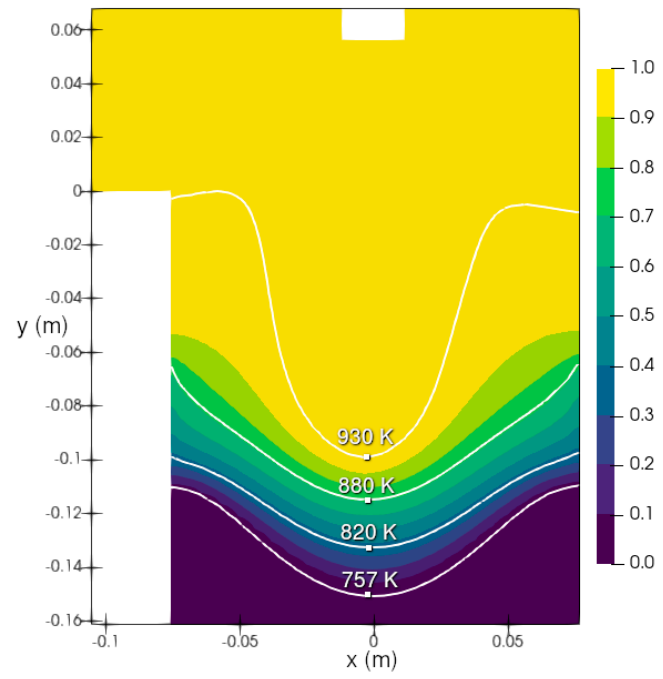

(a)

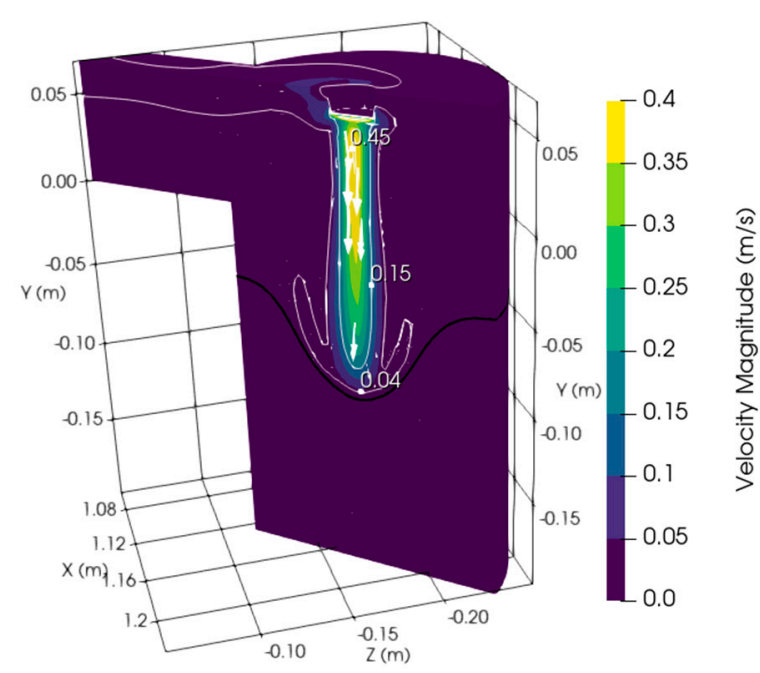

(b)

Figure 7. Resulting solid fraction and temperature profile (a), and fluid flow velocity magnitude (b) in the billet when applying UST directly at the hot-top.

Figure 8a shows the temperature distribution in the launder when UST is applied, and Figure $8 \mathrm{~b}$ when UST is not applied. In both cases, the recirculation pattern resulted in the coldest area being behind the upstream partition, but when UST was applied the amount of stirring resulted in a more even temperature distribution, and a reduced temperature drop between the upstream and downstream partitions. Figure $8 \mathrm{c}$ shows the magnitude of the acoustic streaming induced by the sonotrode, with streaming velocities of the order of $1 \mathrm{~m} / \mathrm{s}$ found directly under the sonotrode. For comparison, Figure $8 \mathrm{~d}$ shows the flow profile when UST was not applied. 
(a)

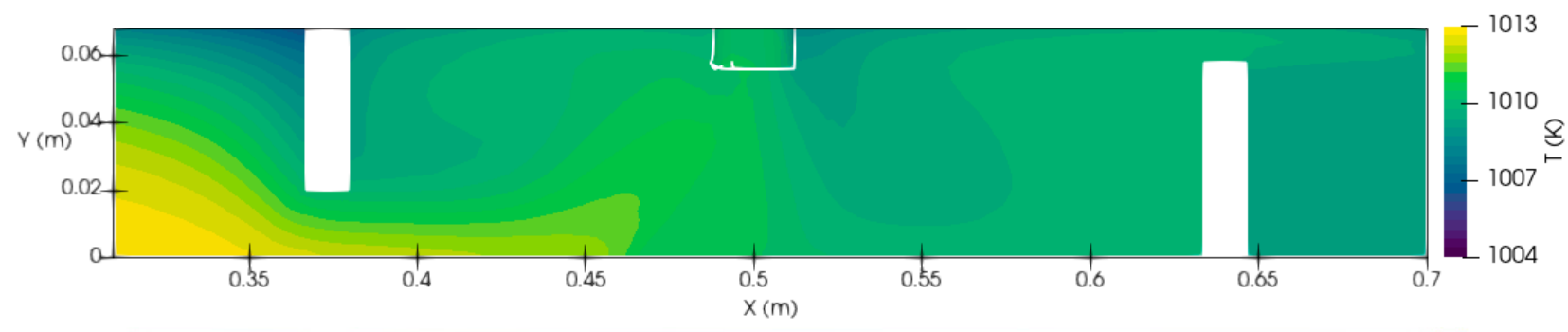

(b)

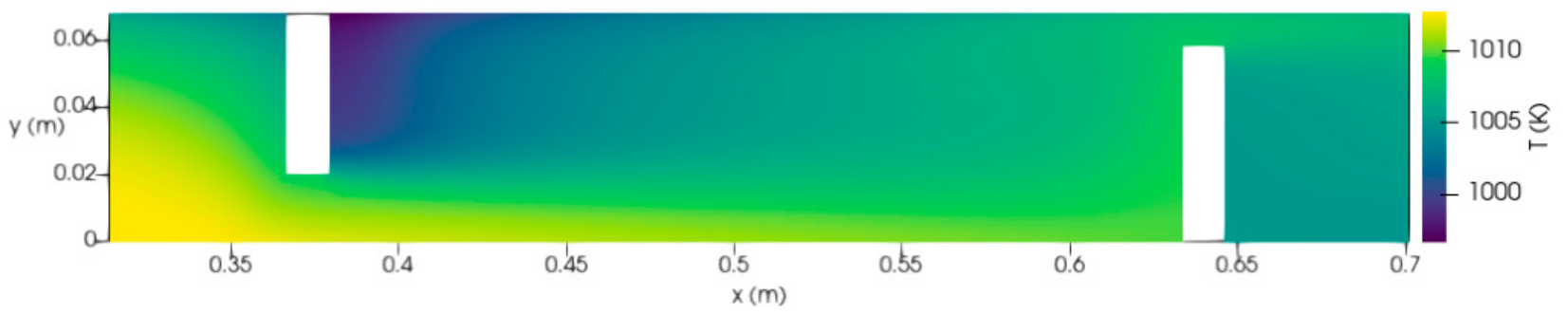

(c)

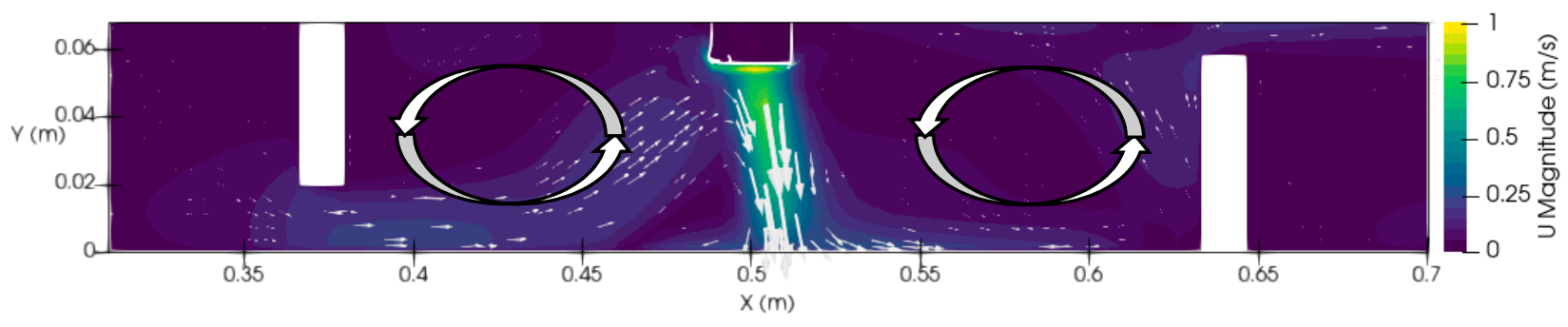

(d)

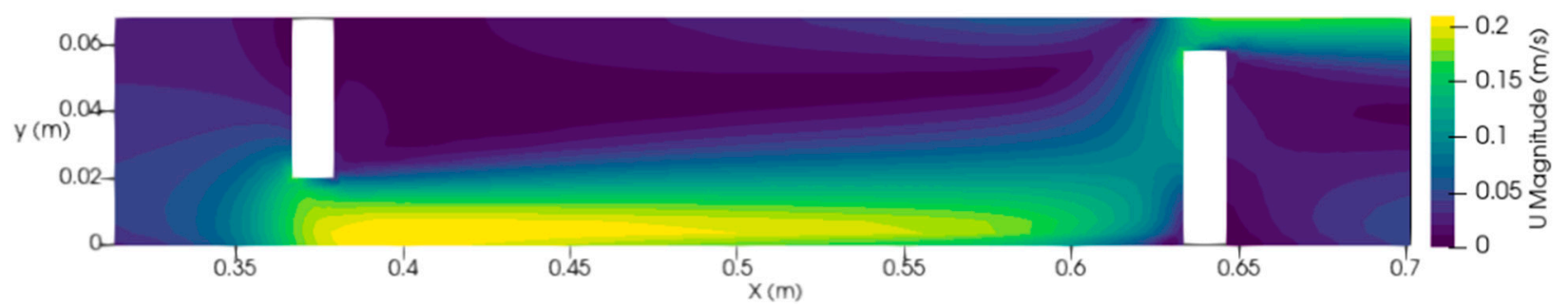

Figure 8. Temperature distribution when UST was applied (a), and without UST (b). Velocity fields when UST was applied (c), and without UST (d). Note the different velocity scales in (c) and (d). Flow from left to right.

The relationship between processing time and grain size was previously established by Atamanenko et al. [30]. It was demonstrated experimentally that grain refinement was not observed in an Al-0.18 mass pct $\mathrm{Zr}-0.07$ Ti alloy not subjected to UST, while upon UST, the grain size reduced from 285 to $180 \mu \mathrm{m}$ after $3 \mathrm{~s}$ of processing, and longer treatment times of 7 and $10 \mathrm{~s}$ further reduced the grain size to 107 and $67 \mu \mathrm{m}$, respectively. Atamanenko et al. [30] clearly established the importance of the active cavitation zone, but only the total processing time was measured, not the residence time in the active zone. The simulation results presented in Figure 9a demonstrate the difference in particle residence time in the active zone when applying UST between the partitions in the launder, or in the hot-top. A total of 10,000 particles were released over a $5 \mathrm{~s}$ interval once the fluid simulation had reached a steady state. Here, residence time is defined simply as the time a refining particle spends suspended in the liquid in a region where the acoustic pressure is above the Blake threshold pressure of $217 \mathrm{kPa}$. Moving the processing into the launder, results in particle recirculation, and a much higher average particle residence time than when processing in the hot-top. The launder case had a mean particle residence time of $0.067 \mathrm{~s}$, with a standard deviation of $0.045 \mathrm{~s}$, compared with a mean of $0.03 \mathrm{~s}$ and a standard deviation of 0.017 for the hot-top. Conversely to Reference [30], the times given relate to the active zone residence time, rather than the total processing time (operation of an ultrasonic transducer), hence the time scales are different. However, it is this active zone residence time that is an important criterion for the efficiency of processing. 

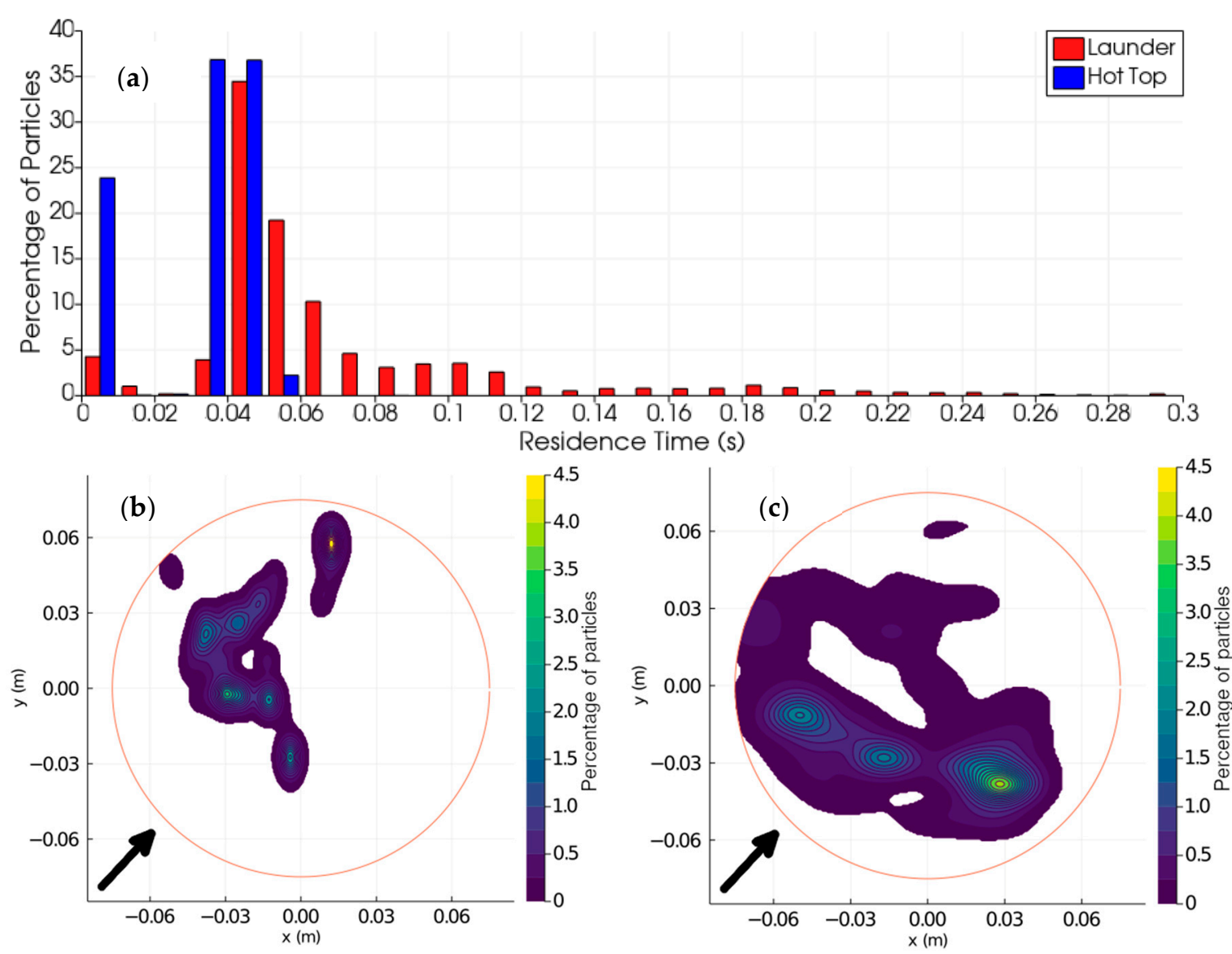

Figure 9. Particle residence time (a), and computed refining particle distributions in the billet through processing in the hot-top (b) and in the launder (c). Arrow on the bottom left indicates the inlet flow direction.

Notably, when processing in the hot-top, many particles do not enter the processing region at all, which could indicate that processing in the launder can activate more refining particles, which then ultimately nucleate aluminium grains and refine the microstructure in the solidifying volume.

The distribution of particles in the resulting billet could be linked with the grain refinement observed in the billet cross-section from the experiments, which were obtained with the experimental procedure mentioned in Section 2. The modelled particle distribution demonstrates that in the case of UST in the hot-top (Figure 9b) the particles were predominantly located in the center of the billet, and near the outside wall opposite from the inlet location. Meanwhile, a much more uniform distribution was observed in the case of UST in the launder, given in Figure 9c. This appeared to correlate with the grain size measurements found in the experiments, as seen in Figure 10. When processing in the hot-top, grain refinement was mostly observed in the center of the billet, with increasing grain size at the inner middle $(20 \mathrm{~mm})$, outer middle $(40 \mathrm{~mm})$, and surface locations. Meanwhile when processing in the launder, a higher degree of grain refinement was observed throughout the cross section of the billet. A qualitatively similar behavior has also been reported in previous experiments [31], in which grain refinement was observed across the billet when UST was applied in a launder, but significant variation in grain size was observed when UST was performed in the hot-top. 


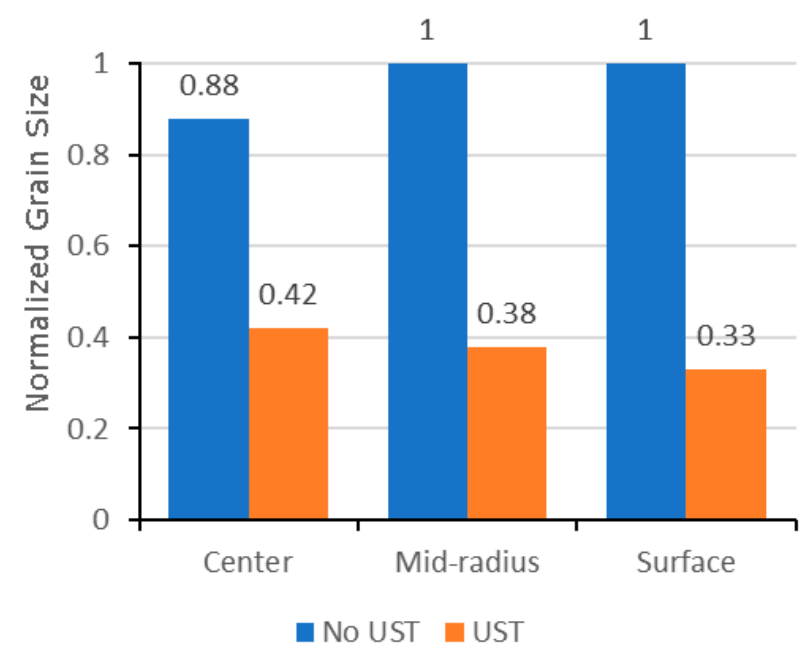

(a)

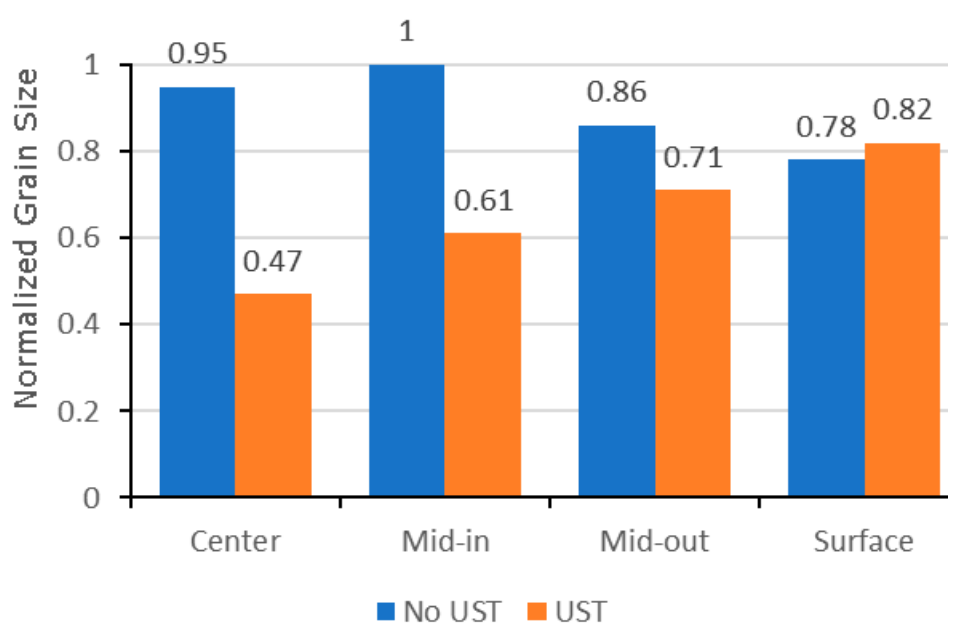

(b)

Figure 10. Normalised grain size in experiments before and after processing, for the launder UST (a) and hot-top UST (b).

\section{Conclusions}

This work has attempted the first numerical comparison between the effects of applying ultrasonic treatment in the hot-top, as is conventionally performed, and the application of UST directly in the launder. Experiments were also carried out, and refining particle statistics from the numerical simulation were shown to correlate with the microstructure grain analysis from experiments.

This demonstrates the potential benefits of moving the UST processing upstream into the launder, at which point melt-flow management systems (partitions) can then be used to further increase residence time, activating more refining particles, and improving the microstructure in the final billet. The effects of including partitions and increasing the operating temperature for UST in the launder have been suggested to be beneficial in previous works [10].

In the current work, only the distribution of dispersed refining particles was considered, but, in reality, these particles are also fragmented or deagglomerated by the shock waves emitted from cavitation. In future work, extending the model to include particle fragmentation and deagglomeration with a range of particle sizes will allow for, not only improved distribution modelling, but also a better approximation of grain size. Furthermore, while our results show that processing in the launder can lead to more dispersed particles, the launder geometry has yet to be optimised and this also needs to be undertaken.

Author Contributions: Conceptualization, C.B. and T.S.; methodology, C.B., T.S. and G.D.; validation, C.B. and T.S.; investigation, C.B., T.S., D.G.E., I.S. and D.R.; writing-original draft preparation, C.B., T.S. and G.D.; writing-review and editing, C.B., T.S., G.D., K.P., D.G.E. and I.T.; supervision, K.P., D.G.E. and I.T.; project administration, K.P., D.G.E. and I.T.; funding acquisition, K.P., D.G.E. and I.T. All authors have read and agreed to the published version of the manuscript.

Funding: This research was funded by EPSRC (UK), under the UltraMelt 2 project, with grant numbers EP/R011001/1, EP/R011044/1, and EP/R011095/1.

Data Availability Statement: Not applicable.

Conflicts of Interest: The authors declare no conflict of interest.

\section{References}

1. Li, S.; Sadayappan, K.; Apelian, D. Role of Grain Refinement in the Hot Tearing of Cast Al-Cu Alloy. Met. Mater. Trans. A 2013, 44, 614-623. [CrossRef]

2. Murty, B.S.; Kori, S.A.; Chakraborty, M. Grain refinement of aluminium and its alloys by heterogeneous nucleation and alloying. Int. Mater. Rev. 2002, 47, 3-29. [CrossRef] 
3. Eskin, G.I.; Eskin, D.G. Ultrasonic Treatment of Light Alloy Melts, 2nd ed.; CRC Press: Boca Raton, FL, USA, 2014.

4. Pericleous, K.; Bojarevics, V.; Djambazov, G.; Dybalska, A.; Griffiths, W.D.; Tonry, C. Contactless Ultrasonic Cavitation in Alloy Melts. Materials 2019, 12, 3610. [CrossRef] [PubMed]

5. Kaldre, I.; Bojarevičs, A.; Grants, I.; Beinerts, T.; Kalvāns, M.; Milgrāvis, M.; Gerbeth, G. Nanoparticle dispersion in liquid metals by electromagnetically induced acoustic cavitation. Acta Mater. 2016, 118, 253-259. [CrossRef]

6. Leighton, T. The Acoustic Bubble, 1st ed.; Academic Press: London, UK, 1997; Volume 10, pp. 149-156.

7. Flannigan, D.J.; Suslick, K.S. Plasma formation and temperature measurement during single-bubble cavitation. Nat. Cell Biol. 2005, 434, 52-55. [CrossRef]

8. Tzanakis, I.; Eskin, D.; Georgoulas, A.; Fytanidis, D. Incubation pit analysis and calculation of the hydrodynamic impact pressure from the implosion of an acoustic cavitation bubble. Ultrason. Sonochem. 2014, 21, 866-878. [CrossRef]

9. Zhang, L.; Li, X.; Li, R.; Jiang, R.; Zhang, L. Effects of high-intensity ultrasound on the microstructures and mechanical properties of ultra-large $2219 \mathrm{Al}$ alloy ingot. Mater. Sci. Eng. A 2019, 763, 138154. [CrossRef]

10. Subroto, T.; Eskin, D.G.; Beckwith, C.; Skalicky, I.; Roberts, D.; Tzanakis, I.; Pericleous, K. Structure Refinement Upon Ultrasonic Melt Treatment in a DC Casting Launder. JOM 2020, 72, 4071-4081. [CrossRef]

11. Trujillo, F.J. A strict formulation of a nonlinear Helmholtz equation for the propagation of sound in bubbly liquids. Part I: Theory and validation at low acoustic pressure amplitudes. Ultrason. Sonochem. 2018, 47, 75-98. [CrossRef] [PubMed]

12. Swaminathan, C.R.; Voller, V.R. A general enthalpy method for modeling solidification processes. Met. Mater. Trans. A 1992, 23, 651-664. [CrossRef]

13. Lebon, G.B.; Tzanakis, I.; Djambazov, G.; Pericleous, K.; Eskin, D. Numerical modelling of ultrasonic waves in a bubbly Newtonian liquid using a high-order acoustic cavitation model. Ultrason. Sonochem. 2017, 37, 660-668. [CrossRef]

14. Keller, J.B.; Miksis, M. Bubble oscillations of large amplitude. J. Acoust. Soc. Am. 1980, 68, 628-633. [CrossRef]

15. Lebon, G.B.; Salloum-Abou-Jaoude, G.; Eskin, D.; Tzanakis, I.; Pericleous, K.; Jarry, P. Numerical modelling of acoustic streaming during the ultrasonic melt treatment of direct-chill (DC) casting. Ultrason. Sonochem. 2019, 54, 171-182. [CrossRef]

16. Lebon, G.B.; Tzanakis, I.; Pericleous, K.; Eskin, D. Experimental and numerical investigation of acoustic pressures in different liquids. Ultrason. Sonochem. 2018, 42, 411-421. [CrossRef] [PubMed]

17. Trujillo, F.J. A strict formulation of a nonlinear Helmholtz equation for the propagation of sound in bubbly liquids. Part II: Application to ultrasonic cavitation. Ultrason. Sonochem. 2020, 65, 105056. [CrossRef]

18. Chang, S.; Stefanescu, D.M. A model for macrosegregation and its application to Al-Cu castings. Met. Mater. Trans. A 1996, 27, 2708-2721. [CrossRef]

19. Bounds, S.; Morán, G.; Pericleous, K.; Cross, M.; Croft, T.N. A computational model for defect prediction in shape castings based on the interaction of free surface flow, heat transfer, and solidification phenomena. Met. Mater. Trans. A 2000, 31, 515-527. [CrossRef]

20. Baserinia, A.R.; Ng, H.; Weckman, D.C.; Wells, M.A.; Barker, S.; Gallerneault, M. A Simple Model of the Mold Boundary Condition in Direct-Chill (DC) Casting of Aluminum Alloys. Met. Mater. Trans. A 2012, 43, 887-901. [CrossRef]

21. Weckman, D.C.; Niessen, P. A numerical simulation of the D.C. continuous casting process including nucleate boiling heat transfer. Met. Mater. Trans. A 1982, 13, 593-602. [CrossRef]

22. Haider, A.; Levenspiel, O. Drag coefficient and terminal velocity of spherical and nonspherical particles. Powder Technol. 1989, 58, 63-70. [CrossRef]

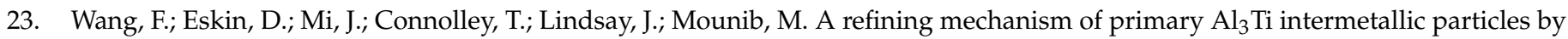
ultrasonic treatment in the liquid state. Acta Mater. 2016, 116, 354-363. [CrossRef]

24. Villars, P.; Cenzual, K. $\mathrm{Al}_{3} \mathrm{Zr}\left(\mathrm{ZrAl}_{3} \mathrm{Rt}\right)$ Crystal Structure: Datasheet from "PAULING FILE Multinaries Edition-2012". Springer \& Materials Phases Data System (MPDS), Switzerland \& National Institute for Materials Science (NIMS), Japan. Available online: https://materials.springer.com/isp/crystallographic/docs/sd_1201137 (accessed on 27 January 2021).

25. Subroto, T.; Eskin, D.G.; Beckwith, C.; Tzanakis, I.; Djambazov, G.; Pericleous, K. Improving Ultrasonic Melt Treatment Efficiency Through Flow Management: Acoustic Pressure Measurements and Numerical Simulations. In Light Metals; Springer: Cham, Switzerland, 2020; pp. 981-987.

26. Tsitouras, C. Runge-Kutta pairs of order 5(4) satisfying only the first column simplifying assumption. Comput. Math. Appl. 2011, 62, 770-775. [CrossRef]

27. Tonry, C.E.H.; Bojarevics, V.; Djambazov, G.; Pericleous, K. Contactless Ultrasonic Treatment in Direct Chill Casting. JOM 2020, 72, 4082-4091. [CrossRef]

28. Mettin, R. Bubble Structures in Acoustic Cavitation; Bubble and Particle Dynamics in Acoustic Fields: Modern Trends and Applications; Research Singpost: Thiruvananthapuram, India, 2005; pp. 1-36.

29. Li, Y.; Li, H.; Katgerman, L.; Du, Q.; Zhang, J.; Zhuang, L. Recent advances in hot tearing during casting of aluminium alloys. Prog. Mater. Sci. 2021, 117, 100741. [CrossRef]

30. Atamanenko, T.V.; Eskin, D.G.; Zhang, L.; Katgerman, L. Criteria of Grain Refinement Induced by Ultrasonic Melt Treatment of Aluminum Alloys Containing Zr and Ti. Met. Mater. Trans. A 2010, 41, 2056-2066. [CrossRef]

31. Zhang, L. Ultrasonic Processing of Aluminum Alloys. Ph.D. Thesis, Delft University of Technology, Delft, The Netherlands, 2013. [CrossRef] 